\title{
PRODUÇÃO DE ALDEÍDOS A PARTIR DO CULTIVO HETEROTRÓFICO DE Phormidium autumnale COM MONOSSACARÍDEOS COMO FONTE DE CARBONO EXÓGENA
}

\author{
A. S. FERNANDES ${ }^{1}$, A. B. SANTOS ${ }^{1}$, G. P. NOGARA ${ }^{1}$, R. WAGNER ${ }^{1}$, E. JACOB- \\ LOPES $^{1}$ e L. Q. ZEPKA ${ }^{1}$ \\ ${ }^{1}$ Universidade Federal de Santa Maria \\ E-mail para contato: 1qz@pq.cnpq.br
}

\begin{abstract}
RESUMO - A elucidação da fase gasosa do sistema de cultivo heterotrófico é importante na consolidação da tecnologia para a exploração industrial. O objetivo do trabalho foi avaliar a formação de aldeídos em biorreatores heterotróficos da cianobactéria Phormidium aumtunale, utilizando duas fontes glicose e frutose como fonte de carbono exógena. Os experimentos foram realizados em biorreator descontínuo com aeração contínua e ausência de luminosidade. Os compostos orgânicos voláteis foram isolados por microextração em fase sólida no headspace (fibra DVB/Car/PDMS), analisados por cromatografia gasosa acoplada a um detector de massas (SPME-GC-MS). A amostragem foi realizada a cada 24 horas no período de 7 dias correspondendo as fases de crescimento. Ao longo do experimento foram detectados 10 aldeídos, com destaque para o 3-metilbutanal com uma produção total de $356,42 \mu \mathrm{g} \cdot \mathrm{mg}_{\text {biomassa }}{ }^{-1}$.
\end{abstract}

\section{INTRODUÇÃO}

Considerando a grande biodiversidade e os recentes desenvolvimentos na engenharia genética, as microalgas representam uma das fontes mais promissoras para novos bioprodutos, sendo essas um conjunto diversificado de organismos com uma grande diversidade na composição química. Por esses motivos tornam-se extremamente atraentes para bioprospecção e exploração potencial como fontes comerciais de uma grande variedade de biomoléculas para novas fontes de produtos químicos e outros produtos de interesse comercial incluindo metabólitos secundários como os compostos voláteis (BOROWITZKA, 2013 e HARUN et al, 2010).

A produção autotrófica é atualmente a mais explorada, em função do metabolismo preferencial fotossintético. Porém a habilidade das microalgas em cultivos heterotróficos utilizando substratos orgânicos para manutenção da existência representa uma alternativa no direcionamento de obtenção de compostos não obtidos nos cultivos convencionais fotossintéticos, além de vantagens como a simplificação de operações unitárias do processo (PEREZ-GARCIA et al, 2011; MINERDI et al, 2009; FAY, 1983). Este tipo de produção é suportada por carboidratos como glicose, frutose e sacarose (HU et al, 2013). 
Embora venha sendo estudado largamente as frações de metabolitos não voláteis, observa-se também que o rendimento em microalga não satisfaz completamente o balanço de carbono total do sistema sugerindo que parte do balanço está direcionado para produção de compostos orgânicos voláteis. No entanto, há pouca informação disponível sobre a biogeração de aromas a partir destes microrganismos (JACOB-LOPES, 2010).

Estes compostos orgânicos voláteis pertencem a várias classes químicas (aldeídos, álcoois, ésteres, terpenos e compostos enxofrados) e são caracterizados pelo baixo peso molecular e a capacidade de interagir com receptores olfativos (MINERDI, 2009).

O uso integral da fração volátil da biomassa microalgal pode representar um avanço em relação ao fornecimento de insumos de grande volume aos mais diferentes tipos de indústria. Um exemplo de aplicação é utilizar aldeídos obtidos a partir de micro-organismos na indústria de aromas. Nesse sentido, existe uma demanda por pesquisas prospectivas da fração volátil de biorreatores microalgais, assim como a elucidação das vias metabólicas da formação desses compostos. O objetivo deste estudo foi avaliar a formação de aldeídos em biorreatores heterotróficos da cianobactéria Phormidium aumtunale, utilizando duas fontes glicose e frutose como fonte de carbono exógena.

\section{MATERIAL E MÉTODOS}

A cianobactéria utilizada foi a Phormidium autumnale, isolada do Deserto Cuatro Sienegas no México (26⒌ ${ }^{\prime} \mathrm{N} 102^{\circ} 03 \mathrm{~W}$ ). As culturas reservas foram mantidas e propagadas em agar-agar solidificado (20 g.. - $^{-1}$ ) com meio sintético BG11 (Rippka et al, 1979). As condições de manutenção usadas foram $25^{\circ} \mathrm{C}$ e intensidade luminosa constante de 1 klux.

Os experimentos foram realizados em um reator de coluna de bolhas. O sistema foi construído de vidro de borosilicato com diâmetro externo de $12,5 \mathrm{~cm}$ e altura de $16 \mathrm{~cm}$, com razão altura/diâmetro proporção igual a 1,28 e um volume nominal de 2,0L. O sistema de dispersão do reator consistiu em um difusor de ar com 2.5 centímetros de diâmetro localizado no interior do bioreator. O fluxo de ar controlado pelo medidor de fluxo (Kl-Key Instruments ${ }^{\circledR}$, Trevose, PA, EUA) e a entrada de ar e a saída de gases serão filtrados com unidades filtrantes constituídas de membrana de polipropileno, com um diâmetro de poro de $0,22 \mu \mathrm{m}$ e o diâmetro total de de 50 mm (Millex FG ${ }^{\circledR}$, Billerica, MA, EUA).

O biorreator, incluindo unidades filtrantes, foram esterilizados em autoclave a $121^{\circ} \mathrm{C}$ durante 40 minutos. Os experimentos foram realizados em biorreatores em regime de batelada, alimentado com $2,0 \mathrm{~L}$ de meio de cultura. As condições experimentais foram as seguintes: concentração inicial do inoculo de $100 \mathrm{mg} / \mathrm{L}$, a temperatura de $26^{\circ} \mathrm{C}, \mathrm{o} \mathrm{pH}$ ajustado para 7,6, aeração de 0,1 VVM (volume de ar por volume de meio por minuto) em ausência de luz. A concentração de frutose e de glicose foram ajustadas a concentração de 12,5 g.L $\mathrm{L}^{-1}$.

Os compostos voláteis formados no bioprocesso foram isolados pela técnica de microextração em fase sólida aplicada em headspace (HS-SPME). A amostragem realizada no tempo zero e a cada $24 \mathrm{~h}$ durante o crescimento celular. A fibra de SPME de revestimento misto empregada foi a DVB/Car/PDMS $(50 / 30 \mu \mathrm{m} \times 20 \mathrm{~mm}$, Supelco Bellefonte, PA, USA), 
pré-condicionada conforme as recomendações fornecidas pelo fabricante. A temperatura de extração de $40^{\circ} \mathrm{C}$, com um tempo de equilíbrio de 5 minutos, após exposição da fibra por 45 minutos. A análise dos compostos voláteis foi realizada em um cromatógrafo a gás acoplado a um espectrômetro de massas (GC/MS Shimadzu QP-2010 Plus). As separações cromatográficas em coluna Chrompack WAX 52-CB $(60 \mathrm{~m} \times 0,25 \mathrm{~mm}$ d.i. $\times 0,25 \mu \mathrm{m}$ de diâmetro de fase estacionária). $\mathrm{O}$ gás de arraste utilizado foi o hélio com vazão constante de 1,6 mL.min ${ }^{-1}$. A temperatura inicial da coluna de $35^{\circ} \mathrm{C}$, permanecendo por 5 minutos, após elevada até $220^{\circ} \mathrm{C}$ com gradiente de temperatura de $5^{\circ} \mathrm{C} / \mathrm{min}$, mantendo-se isotermicamente por 5 minutos. A interface $\mathrm{GC} / \mathrm{MS}$ e da fonte de ionização foram mantidos a $250^{\circ} \mathrm{C}$. O detector de massas foi operado no modo de ionização por elétrons, com feixe de elétrons a $+70 \mathrm{eV}$. O analisador de massas do tipo quadrupolos foi utilizado no modo de varredura na faixa de 35 a $350 \mathrm{~m} / \mathrm{z}$. Os compostos foram identificados primeiramente por comparação dos seus espectros de massa com os do banco de dados espectral da própria biblioteca do GC-MS (NIST MS Search 2.0). A identificação foi confirmada por comparação dos Índices de Retenção Linear calculados.

\section{RESULTADO E DISCUSSÃO}

Os aldeídos produzidos na fração volátil do cultivo heterotrófico de Phormidium autumnale em todos os experimentos foram identificados e quantificados e estão descritos na Tabela 1. Foram produzidos 3 diferentes aldeídos ao longo de 144 horas de tempo de residência no cultivo com glicose e 7 diferentes aldeídos no cultivo com frutose.

Tabela 1 - Aldeídos produzidos na fração volátil do cultivo heterotrófico de Phormidium autumnale com glicose e frutose.

\begin{tabular}{|c|c|c|c|c|c|c|}
\hline & Composto & $\begin{array}{l}\text { Índice de } \\
\text { Retenção }\end{array}$ & $\begin{array}{c}\text { Descritor de } \\
\text { odor }^{\mathrm{a}}\end{array}$ & $\begin{array}{c}\text { Oh } \\
(\mu \mathrm{g} / \mathrm{mg})\end{array}$ & $\begin{array}{c}96 \mathrm{~h} \\
(\mu \mathrm{g} / \mathrm{mg})\end{array}$ & $\begin{array}{c}144 \mathrm{~h} \\
(\mu \mathrm{g} / \mathrm{mg})\end{array}$ \\
\hline 1 & acetaldeído & 648 & fruta, maçã & nd & 10,5 & 1,6 \\
\hline 2 & isobutiraldeído & 821 & $\begin{array}{l}\text { pungente, } \\
\text { malte, verde }\end{array}$ & nd & 0,4 & 0,1 \\
\hline 3 & butanal & 832 & $\begin{array}{l}\text { pungente, } \\
\text { verde }\end{array}$ & nd & 0,4 & 0,1 \\
\hline 4 & 2-metilbutanal & 896 & $\begin{array}{l}\text { cacau, } \\
\text { amêndoa }\end{array}$ & nd & 0,2 & 0,6 \\
\hline 5 & 3-metilbutanal & 910 & malte & nd & 0,6 & 2,2 \\
\hline 6 & hexanal & 1083 & grama, gordura & 4,7 & 5,0 & 1,8 \\
\hline 7 & decanal & 1484 & $\begin{array}{l}\text { sabão, laranja, } \\
\text { gordura }\end{array}$ & 29,8 & 15,4 & 2,1 \\
\hline 8 & 3-octenal & 1498 & verde & nd & 1,1 & 2,1 \\
\hline 9 & $\beta$-ciclocitral & 1602 & menta & nd & 3,4 & 1,3 \\
\hline 10 & 2,4-nonadienal & 1706 & melância & nd & 1,2 & nd \\
\hline
\end{tabular}

nd: Descritor não detectado

a: De acordo com Acree and Arn (2015) 
Durante o tempo de residência pode-se observar uma diminuição da concentração da maioria dos compostos, exceto três destes que apresentaram um aumento em sua concentração ao final do cultivo (2-metilbutanal, 3-metilbutanal e 3-octenal), porém, o 2,4nonadienal desapareceu ao final do experimento. A Figura 1 apresenta os cromatogramas obtidos por GC/MS do cultivo de Phormidium com monossacarídeos no tempo 96 horas com seus respectivos aldeídos em destaque.

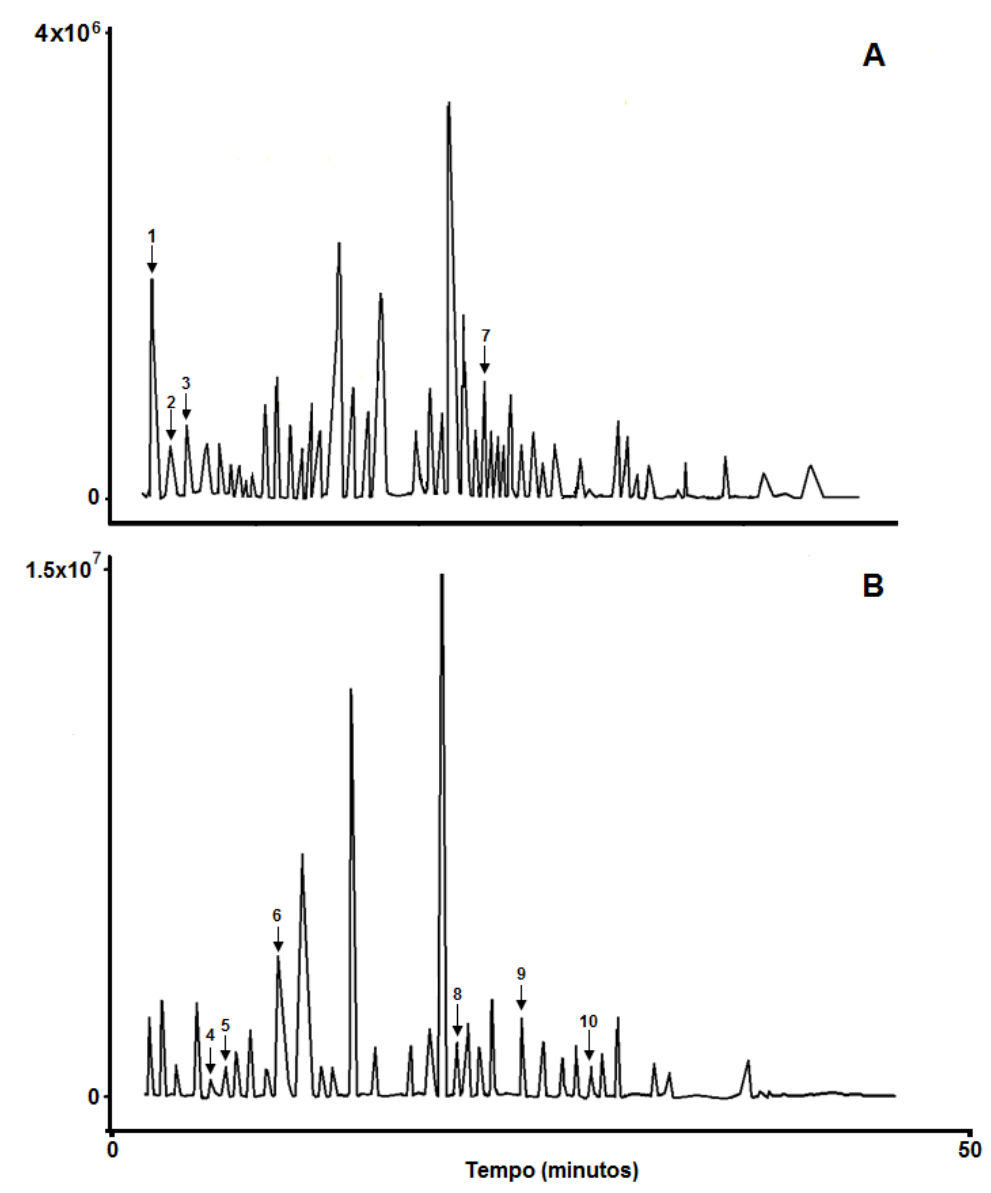

Figura 1. Cromatograma obtido em 96 horas dos cultivos com glicose (A) e frutose (B), com os respectivos aldeídos destacados.

A diminuição da concentração se explica pela rápida conversão de aldeídos em álcoois por uma numerosa quantidade de enzimas endógenas presente em micro-organismos (Kunjapur et al, 2014), justificando a formação de álcoois significativamente alta no final de 144h. Dos componentes identificados em ambos os cultivos, 3-metilbutanal $(2,2 \mu \mathrm{g} / \mathrm{mg})$ destaca-se como majoritário no período de $144 \mathrm{~h}$, seguido pelo 3-octenal $(2,1 \mu \mathrm{g} / \mathrm{mg})$. Conforme Durme et al (2013), os aldeídos de cadeia linear curta são frequentemente derivados de oxidação lipídica química, e as estruturas ramificadas e aldeídos aromáticos são tipicamente formados pela oxidação enzimática de lipídios e proteínas. A produção de $\beta$ ciclocitral pode ser relacionada com a clivagem enzimática de moléculas de $\beta$-caroteno presente na biomassa microalgal, sugerindo a produção pela via biosintética dos terpenos. Esse composto já foi encontrado em microalgas como Chlorella vulgaris e Scenedesmus sp. (Rzama et al, 1995; Durme et al, 2013; Dudareva et al, 2013). 
O esclarecimento das rotas bioquímicas poderá direcionar a produção de biomoléculas específicas do metabolismo microalgal. $\mathrm{O}$ interesse em isolar aldeídos, decorre da utilização destes compostos para fins energéticos e/ou produtos químicos de alto volume de produção.

\section{CONCLUSÃO}

Os resultados indicam que a Phormidium autumnale produziu uma variedade de aldeídos durante o cultivo heterotrófico microalgal suportado por monossacarídeos. A identificação e quantificação destes compostos, além de contribuir para a elucidação de rotas metabólicas desta cianobactéria, apresenta a possibilidade de exploração comercial destes aldeídos para a produção de aromas de interesse da indústria.

\section{REFERÊNCIAS}

ACREE, T., Arn, H. Flavornet and human odor space. www.flavornet.org Last accessed on february 22, 2015.

BOROWITZKA, M. A. High-value products from microalgae their development and commercialisation. Journal of Applied Phycology, 25, 743-756, 2013.

DUDAREVA, N., KLEMPIEN, A., MUHLEMANN, J.K., KAPLAN, I. Biosynthesis, function and metabolic engineering of plant volatile organic coumpounds. New Phytol. 198, 16-32, 2013.

DURME, J.V., GOIRIS, K., WINNE, A., DE COOMAN, L., MUYLAERT, K., 2013. Evaluation of the volatile composition and sensory properties of five species of microalgae. J. Agric. Food Chem. 61, 10881-10890.

FAY, P. The blue-greens (Cyanophyta - Cyanobacteria). Great Britain: 5th Edition, 88 p., 1983.

HARUN, R.; SINGH, M.; FORDE, G. M.; DANQUAH, M. K. Bioprocess engineering of microalgae to produce a variety of consumer products. Renewable and Sustainable Energy Reviews, 14, 1037-1047, 2010.

HU, C. W.; CHUANG, L. T.; YU, P. C.; CHEN, C. N. N. Pigment production by a new thermotolerant microalga Coelastrella sp. F50. Food Chemistry,168, 2071-2078, 2013.

JACOB-LOPES, E.; SCOPARO, C. H. G.; QUEIROZ, M. I.; FRANCO, T. T. Biotransformations of carbon dioxide in photobiorreactors. Energy Conversion and Management, 51, 894-900, 2010.

KUNJAPUR, A.M., TARASOVA, Y., PRATHER, L.J. Synthesis and accumulation of aromatic aldehydes in an engineered strain of Escherichia coli. J. Am. Chem. Soc. 136, 11644-11654, 2014. 
MINERDI, D.; BOSSI, S.; GULLINO, M. L.; GARIBALDI, A. Volatile organic compounds; a potential direct long distance mechanism for antagonistic action of Fusarium oxysporum strain MAS 35. Environmental Microbiology, 11, 844-854, 2009.

PEREZ-GARCIA, O.; ESCALANTE, F. M. E.; DE-BASHAN, L. E.; BASHAN, Y. Heterotrophic cultures of microalgae: Metabolism and potential products. Water Research, 45, 11-36, 2011.

RIPPKA, R., DERUELlES, J., WATERBURY, J.B., HERDMAN, M., STANIER, R.Y. Generic assignments, strain histories and properties of pure cultures of cyanobacteria. $J$. Gen. Microbiol., 111, 1-61, 1979.

RZAMA, A., BENHARREF, A., ARRREGUY, B., DUFOURC, E.J. Volatile compounds of green microalgae grown on reused waste water. Phytochemistry. 38, 1375-1379, 1995. 